\title{
Novel uses of three-parameter logistic models and first-derivative models for the Coronavirus Disease (COVID-19) epidemic in the United States, in three distinct scenarios
}

Bishoy T. Samuel ( $\nabla$ bishoyts@gmail.com )

University of Mississippi Medical Center

\section{Research}

Keywords: Logistic, derivative, COVID-19, United States, t-test, case fatality rate, mortality, inflection, peak, epidemic

Posted Date: August 3rd, 2020

DOl: https://doi.org/10.21203/rs.3.rs-50739/v1

License: (a) (1) This work is licensed under a Creative Commons Attribution 4.0 International License. Read Full License 


\section{Abstract}

Background: Forecasting the current coronavirus disease (COVID-19) epidemic in the United States necessitates novel mathematical models for accurate predictions. This paper examines novel uses of three-parameter logistic models and first-derivative models through three distinct scenarios that have not been examined in the literature as of July 14, 2020.

Methods: Using publicly available data, statistical software was used to conduct a non-linear least-squares estimate to generate a three-parameter logistic model, with a subsequently generated first-derivative model. In the first scenario a logistic model was used to examine the natural log of COVID-19 cases as the dependent variable (versus day number), on July 11 and May 1. Independent $t$-test analyses were used to test comparative coefficient differences across models. In the second scenario, a first-derivative model was derived from a base three-parameter logistic model for April 27 , examining time to peak mortality and decrease in case fatality rate. In the third scenario, a first-derivative model of mortality through July 11 as the dependent variable, versus confirmed cases, was generated to look at case fatality rate relative to increasing cases.

Results: All models generated were statistically significant with $R^{2}>99 \%$. The logistic models in the first scenario best predicted time to growth deceleration in the natural log of cases in the U.S. (slowing of exponential growth), estimated at March 11, 2020. For the May 1 data, independent $t$-test analyses of comparative coefficients across models were useful to track improvements from implemented public health measures. The first-derivative model in the second scenario on April 27, when the epidemic was more controlled, showed peak mortality around April 12-13, with a case fatality rate of $<1,000$ deaths and trending down. The first-derivative model in the third scenario estimated a near-zero case fatality rate to occur at 4 million confirmed cases. It has not been affected by fluctuations in mortality from June 29 through July 11.

Conclusion: Three-parameter logistic models and first-derivative models have utility in predicting time to growth deceleration, and case fatality rates relative to cases. They can objectively assess improvements of implemented epidemiologic measures and have applicable public health safety implications.

\section{Introduction}

In late 2019, a new coronavirus, severe acute respiratory syndrome coronavirus 2 (SARS-CoV-2), was identified in Wuhan, China, and quickly spread to many countries around the world [1]. The virus caused a disease that is now abbreviated as "COVID-19" (coronavirus disease 2019) [2]. The first reported case of COVID-19 in the United States was on January 20, 2020 in the state of Washington, in a patient who had returned from Wuhan, China five days prior [3]. The reporting was confirmed on January 22, 2020 [1].

Since that time the disease has remained an epidemic in the United States. As of July 14, 2020 the Centers for Disease Control and Prevention (CDC) reported $3,296,599$ total cases of COVID-19 in the U.S., and 134,884 total deaths [4].

Several mathematical models have been proposed to forecast the course of COVID-19 in countries around the world, with a few studies discussing the use of the logistic model in countries like China [5, 6] and Saudi Arabia [7]. This type of mathematical model has roots in the classic susceptible-infectious-removed (SIR) epidemiological model [8]. It has also classically been used as an ecological model for population growth to illustrate a population's carrying capacity in limiting the reproductive growth of an organism [9]. An example of this application is in the study of bacterial growth [10]. A literature search yields only one paper published on the use of this model for COVID-19 in the United States as of July 14, 2020, examining the use of a five-parameter logistic growth model to forecast the epidemic's growth [6].

This study aims to examine unique and novel uses of a three-parameter logistic model generated via a non-linear least-squares analysis, with a subsequently generated first-derivative model, in three distinct scenarios. Application of these models through the use of similar scenarios has not been explored in the literature as of July $14,2020$.

The first scenario is to forecast peak cases, determine growth rate, and predict time to growth deceleration by using the natural log of cases as the dependent variable instead of observed case numbers. The second scenario will examine the use of the first-derivative model to predict time to peak mortality and predict trends in the case fatality rate (CFR). The third scenario will examine the combined use of the three-parameter logistic model and the first-derivative model to study daily change in mortality (CFR) relative to case increases.

\section{Methods}

Using Stata/IC 16.0 statistical analysis software, non-linear least-squares estimation was carried out to generate a three-parameter logistic function model for publicly available U.S. COVID-19 case and mortality data from worldometers.info ${ }^{1}[13,14]$. The model form is:

$$
f(x)=\frac{b_{1}}{1+e^{-b_{2}\left(x-b_{3}\right)}}
$$

This paper will examine the use of this model for U.S. COVID-19 data, in three distinct scenarios.

In the first scenario, a three-parameter logistic model was formed using the natural log of U.S. COVID-19 total confirmed cases through July 11 as the dependent variable $y$, with the day number as the independent variable $x$. January 1, 2020 was denoted as day number 1 . The model was represented in the form of: 


$$
\ln y=\frac{b_{1}}{1+e^{-b_{2}\left(x-b_{3}\right)}}
$$

The $b_{1}$ coefficient equaled the natural log of peak cases, the $b_{2}$ coefficient equaled the steepest growth rate of the natural log of cases, and the $b_{3}$ coefficient was the inflection point, which was a midway point calculated as a day number, where the slope (growth rate) of the sigmoid-curve (S-curve) starts decelerating. Independent unpaired $t$-test analysis was used to compare coefficients pairs from several models, to test for statistical significance.

In a second scenario, a first-derivative model was generated following logistic modeling of observed U.S. COVID-19 mortality through April 27,2020 as the dependent variable $y$, with day number as the independent variable $x$. The first-derivative model was in the form of:

$$
f(x)=\frac{d y}{d x}=\frac{b_{1} b_{2} e^{b_{2}\left(x-b_{3}\right)}}{\left(1+e^{b_{2}\left(x-b_{3}\right)}\right)^{2}}
$$

The observed derivative values of the change in mortality versus change in day number were then plotted along with the generated first-derivative model.

In the third and final scenario, a logistic model was generated for U.S. COVID-19 mortality through July 11,2020 as the dependent variable $y$, with U.S. COVID19 total confirmed cases through July 11, 2020 as the independent variable $x$. A subsequently generated first-derivative model was then plotted along with the observed derivative values of these two variables, to show trends in CFR versus increasing number of cases. The methods employed in this third scenario were useful to track CFR changes in U.S. COVID-19 mortality relative to increases in U.S. cases following rapid acceleration after mid-June.

\section{Results}

\section{Scenario 1: Forecasting Peak Cases, Growth Acceleration, and Time to Growth Deceleration}

In the first scenario, the generated three-parameter logistic model is shown in Fig. 1 (Fig. 1). It was generated for data through July 11, 2020, starting with the data point from day 63 , corresponding to March 3 , when the data showed a visually consistent pattern. The coefficient of determination $\left(R^{2}\right)$ value for this model was also 0.9997 , higher than 0.9989 for the model generated from data starting on day 1 . Therefore, all model comparisons in this specific scenario will start from day 63.

The three-parameter logistic model in Fig. 1 was statistically significant with a $p$-value of 0.0000 and $R^{2}$ of 0.9997 . Therefore, greater than $99.9 \%$ of the variability in the natural log of cases can be accounted for by changes in the day number, per this model, and these changes are highly unlikely to be due to chance alone. All generated coefficients $\left(b_{1}\right.$ through $b_{3}$ ) were also statistically significant at $p$-values of 0.000 . Table 1 summarizes the statistics for all model coefficients, including their $95 \%$ confidence intervals $(95 \% \mathrm{Cl})$.

Table 1

Coefficient Statistics for Logistic Model for Cases through July 11

\begin{tabular}{|llllll|}
\hline Coefficient & Value & p-value & Standard Error & Lower 95\% Cl & Upper 95\% Cl \\
\hline$b_{1}$ & 14.5026 & 0.000 & 0.0276518 & 14.44789 & 14.55732 \\
\hline$b_{2}$ & 0.0779727 & 0.000 & 0.0013586 & 0.0752845 & 0.080661 \\
\hline$b_{3}$ & 71.08833 & 0.000 & 0.1929421 & 70.70656 & 71.47010 \\
\hline
\end{tabular}

When the model was run for data up to day 109 , the $b_{1}$ coefficient was 14.24819 ( $p$-value of $0.000,95 \%$ Cl: $[14.01002,14.48636]$ ) and the $b_{3}$ coefficient was 70.86912 ( $p$-value of $0.000,95 \% \mathrm{Cl}$ : $[70.43388,71.30436]$ ). As seen in Table 1 , the $b_{1}$ coefficient for the above model was 14.5026 and the $b_{3}$ coefficient was 71.08833. Compared to much earlier in the pandemic, the most recent model at the time of this writing therefore shows variability in peak case coefficient $\left(b_{1}\right)$ of 0.25441 , which translates to a difference of 446,539 cases when $e^{b_{1 i s}}$ calculated for each model. The variation in the inflection point $\left(b_{3}\right)$ was much more telling, exhibiting less than 1-day variability (0.21921 days) compared to 93 days prior.

Another application of this three-parameter logistic model was employed earlier in the epidemic, when U.S. cases started to flatten due to continued social distancing and proper precautions. Figure 2 shows the generated three-parameter logistic model for U.S. COVID-19 cases through May 1, 2020, with the dashed line denoting the model for three weeks prior to May 1 . Although the models are visually very similar, statistical analysis comparing the $b_{1}$ and $b_{3}$ coefficients from both models showed statistically significant differences between each respective pair. This showed that implemented public health measures at that time of the epidemic yielded statistically detectable differences in total peak cases and time to growth deceleration, three weeks apart, even when not visually apparent. Table 2 shows the coefficient statistics for both models. Table 3 shows the statistically significant results of independent $t$-test analyses comparing $b_{7}$ and $b_{3}$ coefficients from each model. The analyses assumed a null hypothesis $H_{0}$ stating there is no difference between each pair of comparative coefficients, and an alternative hypothesis $H_{a}$ stating that there is a difference, at a desired a-value of 0.05 . 
Table 2

Coefficient Statistics for Logistic Model through May 1, and Three Weeks Prior

\begin{tabular}{|lllllll|}
\hline Model & Coefficient & Value & $p$-value & Standard Error & Lower 95\% Cl & Upper 95\% Cl \\
\hline May 1 & $b_{1}$ & 13.98858 & 0.000 & 0.0315692 & 13.92531 & 14.05184 \\
& $b_{2}$ & 0.0905523 & 0.000 & 0.0009855 & 0.0885773 & 0.0925272 \\
& $b_{3}$ & 70.42847 & 0.000 & 0.0868735 & 70.25437 & 70.60257 \\
\hline Three Weeks Prior & $b_{1}$ & 14.24819 & 0.000 & 0.117196 & 14.01002 & 14.48636 \\
& $b_{2}$ & 0.0871255 & 0.000 & 0.0018367 & 0.0833929 & 0.0908581 \\
& $b_{3}$ & 70.86912 & 0.000 & 0.2141687 & 70.43388 & 71.30436 \\
\hline
\end{tabular}

Table 3

Independent $t$-test analysis results

\begin{tabular}{|ll|}
\hline Coefficient Compared & $p$-value \\
\hline$b_{1}$ & 0.0000 \\
\hline$b_{3}$ & 0.0000 \\
\hline
\end{tabular}

Alternatively, when a three-parameter logistic model was generated for case data two weeks prior to May $1, t$-test analyses between the respective coefficients did not show statistical significance. For example, independent $t$-test analysis comparing the $b_{2}$ coefficients, representing the steepest case growth rates, yielded a $p$-value of 0.1114 . Therefore, the results were not statistically significant, and $H_{0}$ would not be rejected in this case, concluding that there is no difference between both coefficients and that growth rate is stabilizing between models.

\section{Scenario 2: Forecasting Time to Peak Mortality and Case Fatality Rate Trends}

Data for U.S. COVID-19 mortality from April 27 was used as the dependent variable $y$, with day number as the independent variable $x$. A three-parameter logistic model was then generated using a non-linear least-squares estimation method. A subsequently generated first-derivative model was then calculated. In Fig. 3, the first-derivative function is plotted with the observed derivative values for the two variables. This method's main utility was in tracking changes in mortality, or CFR, with progressing days. The overlying first-derivative model predicted time to peak mortality, and also showed the trending CFR. When it was generated on April 27, 2020, the model showed that peak mortality occurred between days 101 to 102 (April 12 to April 13) and that the CFR was trending < 1,000 deaths/day at the time the model was generated, with a forecast for a continued downward trend (which has been true, despite increasing cases as of July 11).

\section{Scenario 3: Forecasting Case Fatality Rate Changes Relative to Accelerated Increase in Cases}

To examine changes in mortality (CFR) relative to the accelerating U.S. COVID-19 case increases since mid-June to date, a combination of a three-parameter logistic model with a subsequent first-derivative model was used. First, a non-linear least squares regression was carried out on U.S. COVID-19 mortality through July 11, 2020 as the dependent variable $y$, with U.S. COVID-19 total confirmed cases through the same date as the independent variable $x$, and a threeparameter logistic model was then generated (Fig. 4). A model with a perfect $R^{2}$ of 1.0000 was achieved when the model was fitted starting from data on day 109, April 20, 2020, when the U.S. was slightly under 1 million COVID-19 cases $(\sim 800,000)$.

The generated three-parameter logistic model was statistically significant at a $p$-value of 0.0000 with a perfect $R^{2}$ of 1.0000 , wherein $100 \%$ of the variance in mortality is predictable from the cases. Table 4 shows the model coefficient statistics.

Table 4

Coefficient Statistics for Logistic Model of Mortality versus Cases through July 11

\begin{tabular}{|llllll|}
\hline Coefficient & Value & p-value & Standard Error & Lower 95\% Cl & Upper 95\% Cl \\
\hline$b_{1}$ & 136643.6 & 0.000 & 247.2954 & 136151.7 & 137135.6 \\
\hline$b_{2}$ & 1960000 & 0.000 & 1130000 & 1940000 & 1990000 \\
\hline$b_{3}$ & 1162495 & 0.000 & 2662.891 & 1157197 & 1167792 \\
\hline
\end{tabular}

A subsequently generated first-derivative model was then calculated for these two variables, to track daily change in mortality (CFR) relative to daily changes in U.S. COVID-19 cases through July 11, 2020. Figure $5^{2}$ shows the generated first-derivative model plotted against the observed derivative data for the two variables.

As the observed data shows, changes in mortality relatively flattened once the U.S. was past 3 million confirmed COVID-19 cases (on July 5, 2020). The model Loading [Math Jax]/jax/output/CommonHTML/jax.js cases of near-zero starting around 4 million confirmed U.S. COVID-19 cases, if current conditions remain constant 
as of July 11. Furthermore, fluctuations in mortality since June 29 have not affected the fit of the generated three-parameter logistic model (which remained with a perfect $R^{2}$ of 1.0000 when generated daily since then), or the subsequent first-derivative model.

\section{Discussion}

In the first scenario, the use of the natural log of U.S. COVID-19 total confirmed cases as the dependent variable, and not the number of observed cases directly, was a novel approach in COVID-19 modeling. This approach was useful in determining the accuracy of the $b_{1}$ and $b_{3}$ coefficients: the peak of the natural log of cases, and the inflection point midway on the S-curve correlating to the day number denoting deceleration in the natural log of cases, respectively. The small variability in both coefficients compared to models from earlier in the epidemic, especially for the inflection point, which was less than 1 day, has pragmatic importance. In a real-world application, this use of a three-parameter logistic model shows highly reliable predictability in the day number representing the point of deceleration for case growth, whether earlier the epidemic or at later points. Estimation of peak cases $\left(b_{7}\right)$ is discussed as a possible limitation later in this paper.

The utility of the independent $t$-test analyses carried out in the first scenario also have practical applications. For example, statistical significance between comparative coefficients from different models indicate that there are improvements in different parameters of the epidemic. On the other hand, lack of statistical significance in the respective coefficients signify that case numbers are stabilizing to a point so as not to show statistically discernible differences in these parameters: peak cases, growth rate, or time to deceleration. Both results signify improvements in the epidemic that are not immediately obvious from the visual models or statistical analyses alone. Therefore, this is one measure to assess the effectiveness of epidemiologic public health measures when they are being adhered to. This also has implications as a tool for public health leaders, to help in their communication with the general public and encourage adherence to proposed public health safety measures.

In the second scenario, a first-derivative model generated from a base three-parameter logistic model was useful as another method to objectively gauge control of the epidemic by forecasting CFR trends, specifically, during times of already-instituted public health measures. This approach would have been useful during a time when cases of the COVID-19 epidemic were beginning to flatten in the United States, such as at the time this scenario's model was generated (April 27). At such time, the general public would be concerned with time until ease of restrictions. This model would have served to give encouraging information that peak mortality had passed and would have had utility in predicting a good future date to begin policy implementations for easing certain restrictions, based on an effective CFR of near-zero. Given the individualistic culture of the United States, such information can serve to help the general public understand that adherence to certain public health safety measures is necessary for a short while longer.

The third and final scenario has high-yield utility at a time when the COVID-19 epidemic in the United States is experiencing accelerated growth, after initially plateauing for a brief interval. With a growing number of Americans not choosing to wear masks, a topic of public debate [11], a model such as this could serve as a tool to guide public health leadership in making individualized epidemiologic recommendations taking into account the non-uniformity of American culture. The first-derivative model in this scenario proved to be a novel approach to examining actual daily changes in mortality versus daily changes in case rates, a marker of the COVID-19 epidemic's impact with continued case increases.

With these models, decreasing CFR relative to increasing total cases of COVID-19 may indicate that aggressive treatments are being utilized readily in hospitalized patients, that those contracting the virus are not dying from it, or that the most vulnerable are being better-protected. For example, it is known that older adults and individuals with certain chronic medical conditions are most susceptible to severe illness or death from COVID-19 [12]. If CFR continues a downwards trend, that may indicate that more young individuals with mild or no symptoms are contracting the virus and not dying from it. On the other hand, if according to this model CFR starts to increase relative to accelerated case increases, then that may indicate that the young and healthy are starting to transmit the virus back to older individuals like parents, relatives, neighbors, etc. This type of conclusion is not only a critical objective measure of the current course of the virus in the population but can also serve to guide public health safety recommendations that are focused, specific, and individualized to help control the epidemic. Such recommendations may target the most vulnerable individuals at risk of death from COVID-19 and would also better take into account the current political and cultural climate in the United States.

\section{Limitations}

The accuracy of three-parameter logistic models in predicting peak cases depends on several factors being constant in the United States, like constant rates of testing, constant rates of infection, constant rates of recovery, and a predictable and constant changing rate of deaths. Any easing of restrictions, enacting new policies or public safety measures, or improvements in testing and/or treatments will affect all these constants, and consequently affect the growth rate and predicted peak cases. This is especially applicable to models that use the actual observed case numbers as the dependent variable, where significant differences in growth rate and peak cases are seen depending on when the model was calculated at different points in the epidemic. A similar limitation is discussed in other papers. In a study using the three-parameter logistic model to forecast COVID-19 cases in China, the authors noted that the model failed to estimate case numbers in the early stages of the epidemic, and was only accurate when there was an apparent maximum being reached in cases [5]. In another study using a five-parameter logistic model to estimate COVID-19 cases in the United States, data was used from March 21 to April 4, estimating a peak of about 800,000 cases [6]. As is evident now by the present number of cases, the model did not accurately predict present case numbers. The authors note that the model's long-term predictability for future new cases may not be accurate, and that it was limited to the data collected over the short interval in the study [6]. The study conducted in this paper found similar limitations as discussed in these two studies $[5,6]$ in the use of the three-parameter logistic models to model COVID-19 data.

Of note, in the three-parameter logistic model study for China [5] the authors tested for heteroskedasticity in the data, and examined residuals, two methods which woro nnt nart of thic nanor $\Delta$ len unlike a different study discussing use of the logistic model study for cases in China [3], this paper did not examine Loading [MathJax]/jax/output/CommonHTML/jax.js 
applicability to other countries.

In the first scenario, the estimated $b_{1}$ coefficient, the peak of the natural log of U.S. COVID-19 cases, is calculated at 14.5026. This gives an estimate of $1,987,921$ peak cases, when $e^{b_{1}}$ is calculated. When the same model was run with the observed case data as the dependent variable the peak cases predicted were $3,414,134$, which is a closer estimate to the present number of U.S. COVID-19 cases as noted in the Introduction. The best utility therefore of the suggested three-parameter logistic model in the first scenario, using the natural log of cases as the dependent variable, would be to estimate the inflection point coefficient $b_{3}$ since it showed minimal variability. This is when the growth of the natural log of cases would start to decelerate and is effectively the same point of deceleration for the exponential growth of the actual observed case data.

The first-derivative models have few limitations, chiefly because they are useful in tracking changes in actual rates, whether in cases, or in mortality, as discussed in the second and third scenarios. Therefore, the peaks of first-derivative models will correlate to the inflection point of their base three-parameter logistic model, the point when rate deceleration begins. Their utility in estimating "zero rate," however, is limited by the same parameters concerning peak cases in logistic models.

\section{Conclusion}

For the U.S. COVID-19 epidemic, three-parameter logistic models have significant utility in predicting changes in growth rate of data, time to growth deceleration, with limitations in predicting peak cases. First-derivative models have similar utility in tracking changes in mortality (CFR) especially relative to increase in active cases. The models also have applicable public health safety and policy implications. Finally, the ultimate utility of these models is that they are repeatable, efficient, and replicable with the ability to compare the statistical significance of coefficients generated from different models at different points in the epidemic.

\section{Declarations}

\section{Acknowledgements}

Not applicable.

Ethics approval and consent to participate

Not applicable

\section{Consent for publication}

Not applicable

\section{Availability of data and materials}

The datasets generated and/or analyzed during the current study are available in the Worldometer and Visual Capitalist repositories, at https://www.worldometers.info/coronavirus/country/us/ and at https://www.visualcapitalist.com/infection-trajectory-flattening-the-covid19-curve/? fbclid=IwAR00ciSFbK60vPwwGBYA6tBLTVdU6eY-WmxF7NuEMAnpnc-B7UZsY2XL7M4.

\section{Competing interests}

The author declares that he has no competing interests.

\section{Funding}

Not applicable.

\section{Author's information}

Dr. Bishoy Samuel completed his M.D. through the University of South Florida College of Medicine in Tampa, Florida in 2009 and finished internal medicine residency in 2012. In his early career he served in various leadership roles including Assistant Chief of Staff at a rural Alabama hospital, and helped secure a grant to implement novel interventions for hypertension and diabetes in the state. He later worked in urban Los Angeles as an outpatient primary- and urgent care physician. In 2019 he enrolled in the University of Mississippi Medical Center Preventive Medicine Residency, to address his growing interests in nutrition, policy, and medical leadership. He is presently completing a Master of Science in Population Health as part of his residency and is also a Healthy Policy Fellow in the Center for Mississippi Health Policy.

\section{Author's contributions}

Bishoy T. Samuel conducted all background and literature searches, compiled all relevant data, conducted all data analysis, and wrote all sections of this manuscript, with no other contributions.

\section{Abbreviations}


CDC: Centers for Disease Control and Prevention

CFR: Case Fatality Rate

Cl: Confidence Interval

COVID-19: Coronavirus disease 2019

$R^{2}$ : Coefficient of determination

SARS-CoV-2: Severe acute respiratory syndrome coronavirus 2

S-curve: sigmoid curve

\section{Footnotes}

1. Worldometers data, at https://www.worldometers.info/coronavirus/country/us/, was current as of June 29,2020 . Daily updates since then were carried out, through July 11 . This dataset starts from February 15 . Worldometers cites that they primarily reference the Centers for Disease Control and Prevention (CDC) for their United States data. In addition, data for days from January 22 through February 14 were obtained from Visual Capitalist, at https://www.visualcapitalist.com/infection-trajectory-flattening-the-covid19-curve/?fbclid=IwAR00ciSFbK60vPwwGBYA6tBLTVdU6eY-

WmxF7NuEMAnpnc-B7UZsY2XL7M4. This latter step was done for completeness of the data, but did not affect any of the models generated, as the models were regressed from data points later in the epidemic, that were part of the main Worldometers data. Visual Capitalist cites that they obtain data from the European Center for Disease Prevention and Control (ECDC).

2. The observed derivative line graph in Figure 5 (red) does not show visual outliers for day 55 , days 57 through 64 , and days 66 through 68 . This was done for visual cohesiveness of the observed line graph in Figure 5. This did not affect the generated first-derivative model (blue), which instead depended on the base three-parameter logistic model derived from the original observed COVID-19 mortality data and observed case data.

\section{References}

1. Harcourt J, Tamin A, Lu X, Kamili S, Sakthivel SK, Murray J, et al. Severe Acute Respiratory Syndrome Coronavirus 2 from Patient with Coronavirus Disease, United States. Emerg Infect Dis. 2020;26(6):1266-1273. https://dx.doi.org/10.3201/eid2606.200516.

2. World Health Organization. Naming the coronavirus disease (COVID-19) and the virus that causes it. 2020. https://www.who.int/emergencies/diseases/novel-coronavirus-2019/technical-guidance/naming-the-coronavirus-disease-(covid-2019)-and-the-virus-thatcauses-it. Accessed 14 Jul 2020.

3. Holshue ML, DeBolt C, Lindquist S, Lofy K, Wiesman J, Bruce H, et al. First Case of 2019 Novel Coronavirus in the Untied States. N Engl J Med 2020; 382:929-936 doi: 1056/NEJMoa2001191.

4. Centers for Disease Control and Prevention. Coronavirus Disease 2019 (COVID-19). 2020. https://www.cdc.gov/coronavirus/2019-ncov/casesupdates/cases-in-us.html. Accessed 14 Jul 2020.

5. Shen CY. Logistic growth modelling of COVID-19 proliferation in China and its international implications. Int J Infect Dis. 2020 Jul; 96 : $582-$ 589.doi: 1016/j.jid.2020.04.085.

6. Chen DG, Chen X, Chen JK. Reconstructing and forecasting the COVID-19 epidemic in the United States using a 5-parameter logistic growth model. Glob Health Res Policy. 2020; 5:25. doi: 1186/s41256-020-00152-5.

7. Alboaneen D, Pranggono B, Alshammari D, Alqahtani N, Alyaffer R. Predicting the Epidemiological Outbreak of the Coronavirus Disease 2019 (COVID-19) in Saudi Arabia. Int J Environ Res Public Health. 2020 Jun 25;17(12):4568. doi: 3390/ijerph17124568.

8. Abou-Ismail A. Compartmental Models of the COVID-19 Pandemic for Physicians and Physician-Scientists. SN Compr Clin Med. 2020 Jun 4: 1-7. doi: 1007/s42399-020-00330-z.

9. Fisher, MR. Population Growth and Regulation. LibreTexts Biology. 2020.

https://bio.libretexts.org/Bookshelves/Ecology/Book\%3A_Environmental_Biology_(Fisher)/04\%3A_Community_Population_Ecology/4.02\%3A_Population Accessed 14 Jul 2020.

10. Fujikawa H, Kai A, Morozumi S. A new logistic model for bacterial growth. Shokuhin Eiseigaku Zasshi. 2003;44(3):155-160. doi:3358/shokueishi.44.155.

11. Godoy, M. Yes, Wearing Masks Helps. Here's Why. NPR. 2020. https://www.npr.org/sections/health-shots/2020/06/21/880832213/yes-wearing-maskshelps-heres-why. Accessed 14 Jul 2020.

12. Centers for Disease Control and Prevention. People Who Need to Take Extra Precautions. In: Coronavirus Disease 2019 (COVID-19). 2020. https://www.cdc.gov/coronavirus/2019-ncov/need-extra-precautions/index.html. Accessed 14 Jul 2020.

13. United States Coronavirus Cases. 2020. https://www.worldometers.info/coronavirus/country/us/. Accessed 11 Jul 2020.

14. Routley, N. Infection Trajectory: See Which Countries are Flattening Their COVID-19 Curve. Visual Capitalist. 2020. https://www.visualcapitalist.com/infection-trajectory-flattening-the-covid19-curve/?fbclid=IwAR3uOBgaf6UGHpunRIIxqyJ5yW7HQB0zCjEVcs-FpYzlbuNNBsY5hBRkbY. Accessed $11 \mathrm{Jul} 2020$. 


\section{Figures}

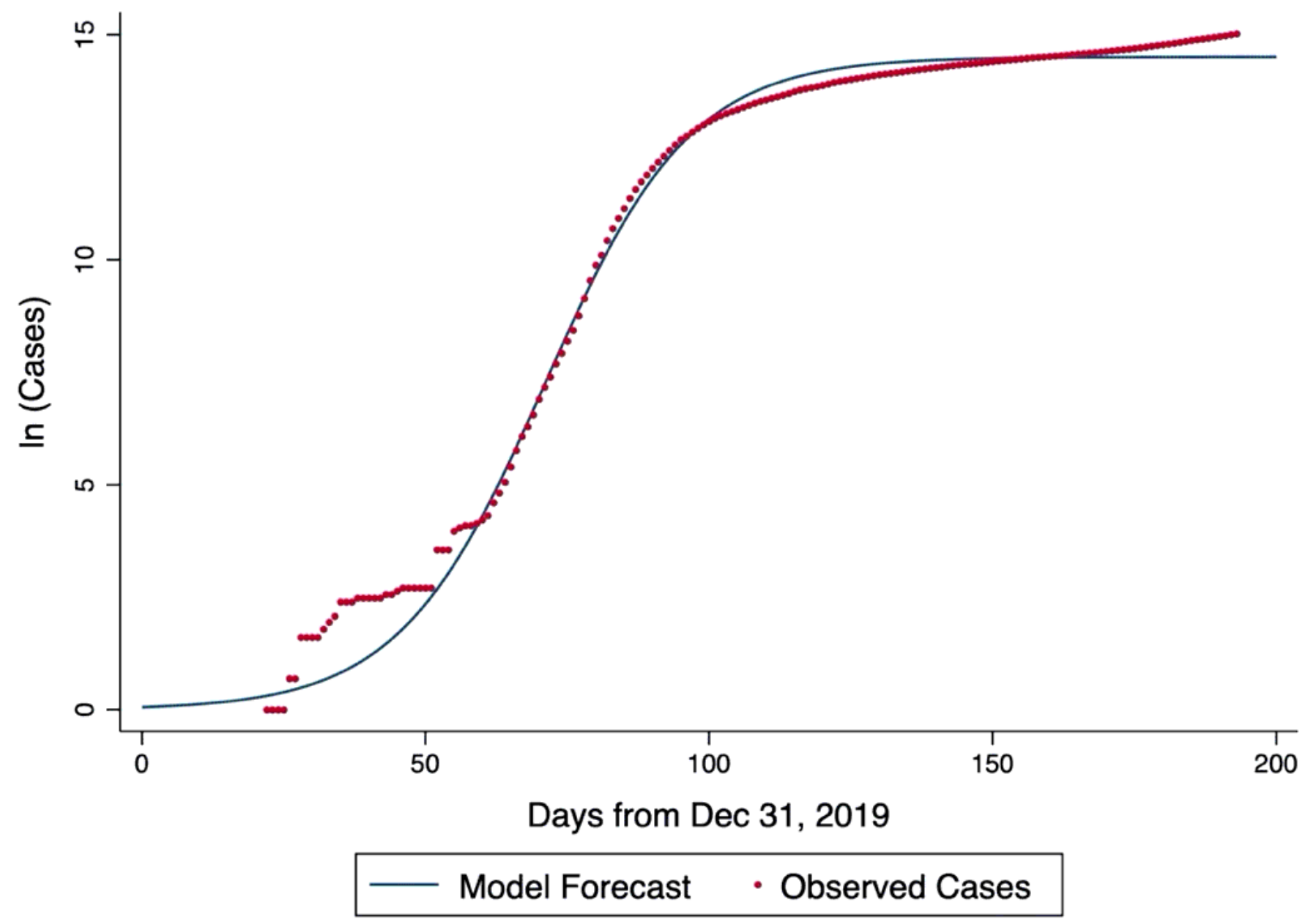

Figure 1

USA COVID-19 Cases: July 11, 2020 


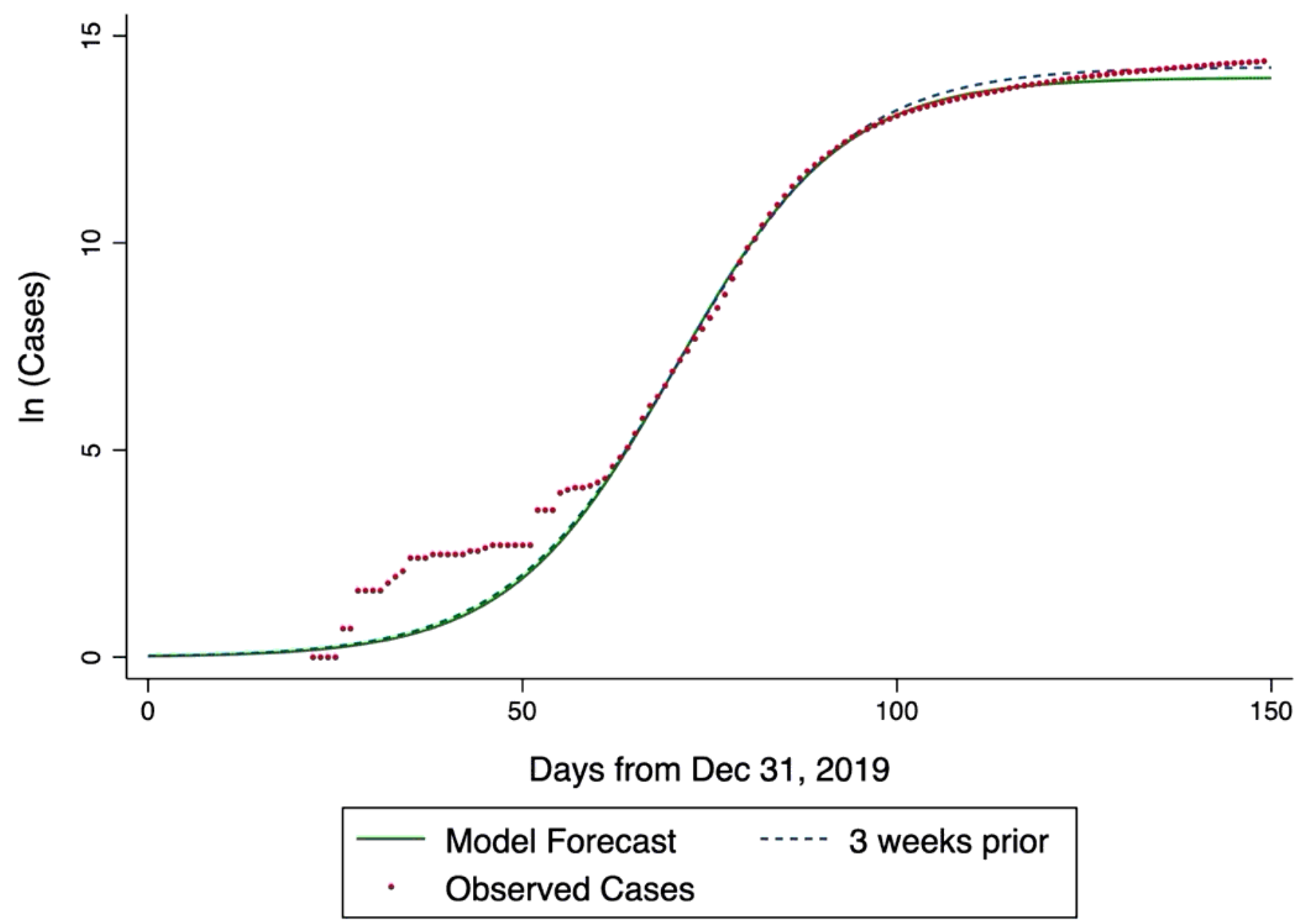

Figure 2

USA COVID-19 Cases: May 1, 2020 


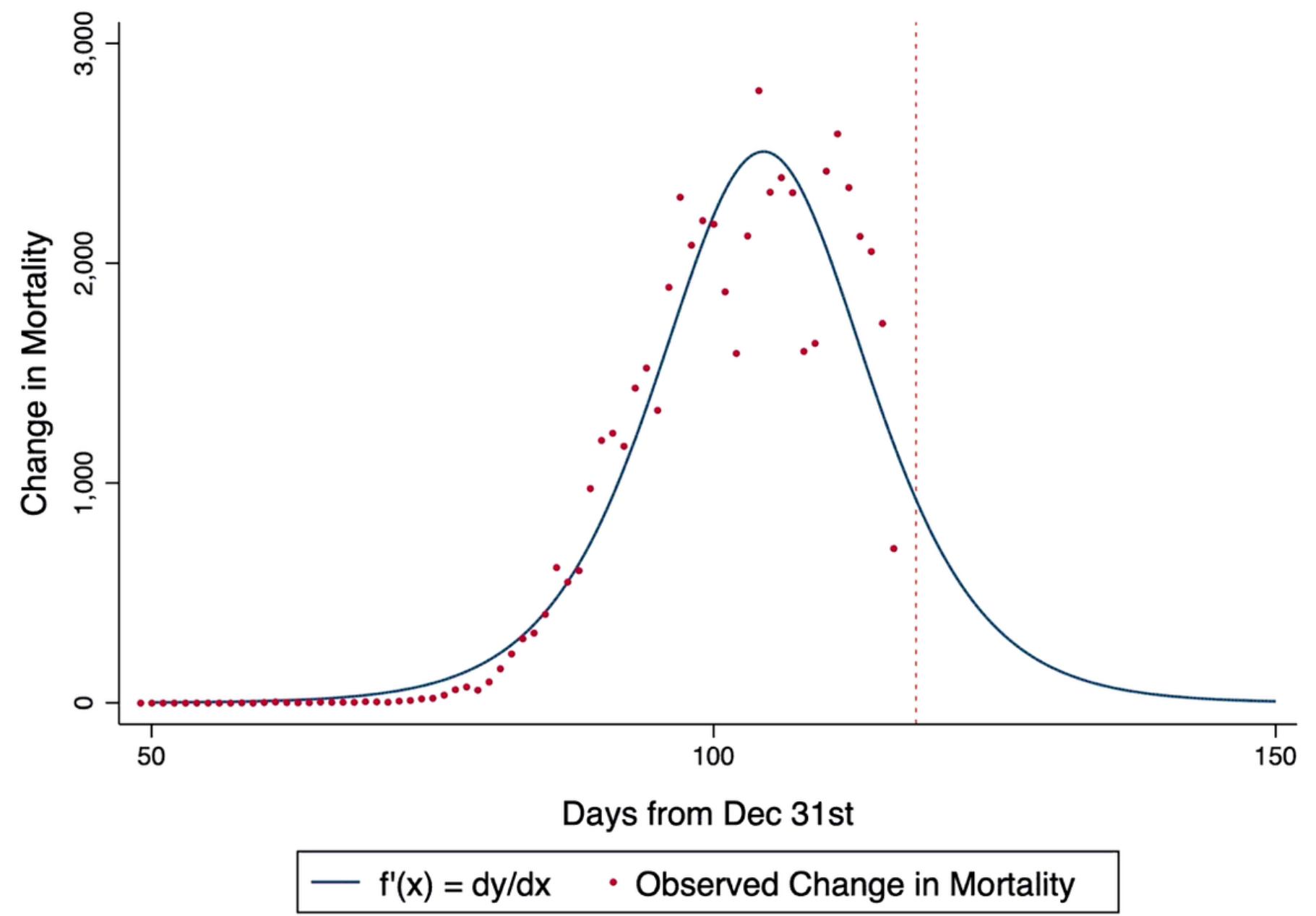

Figure 3

Change in USA COVID-19 Mortality: April 27, 2020 


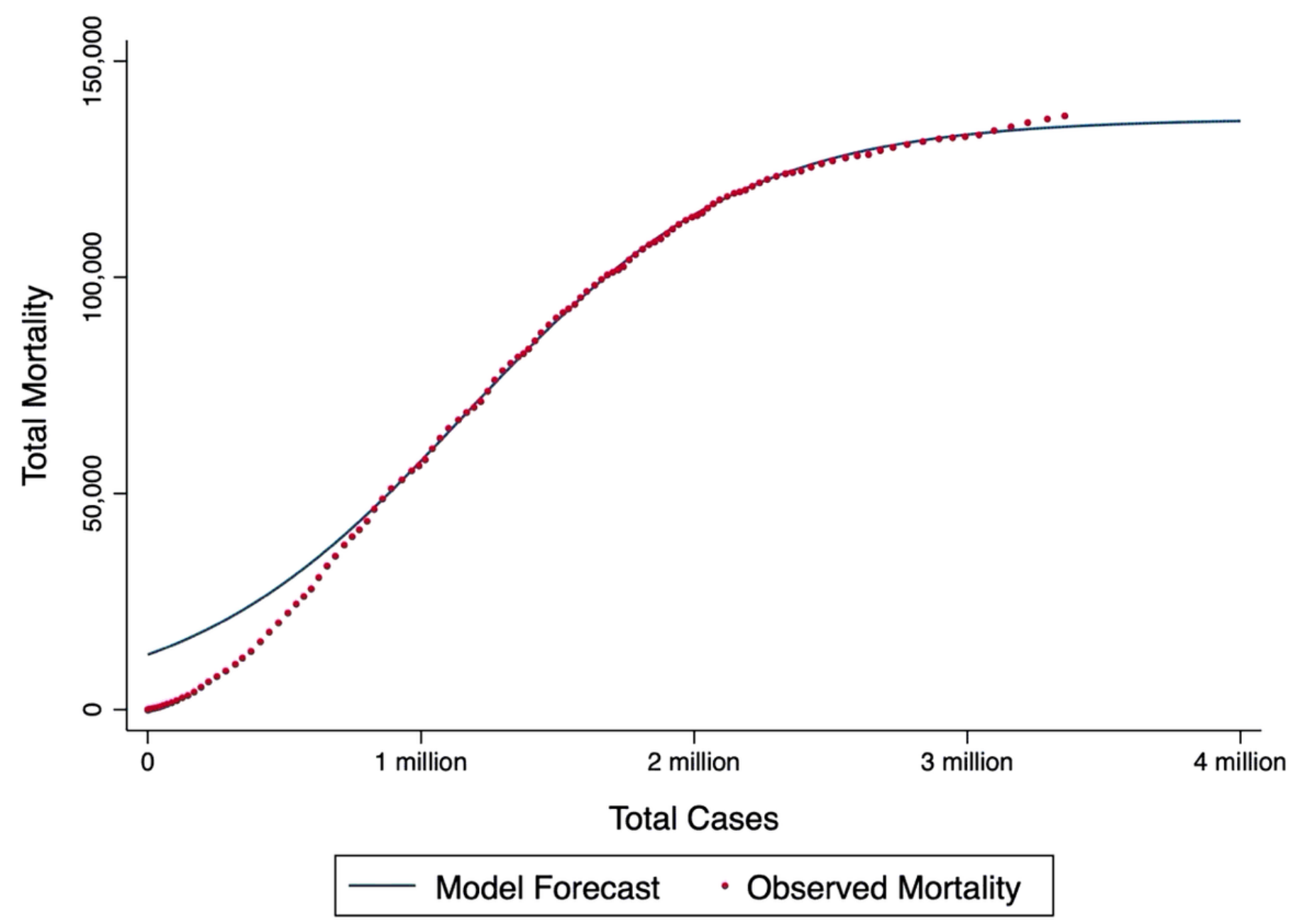

Figure 4

USA COVID-19 Mortality versus Cases: July 11, 2020 


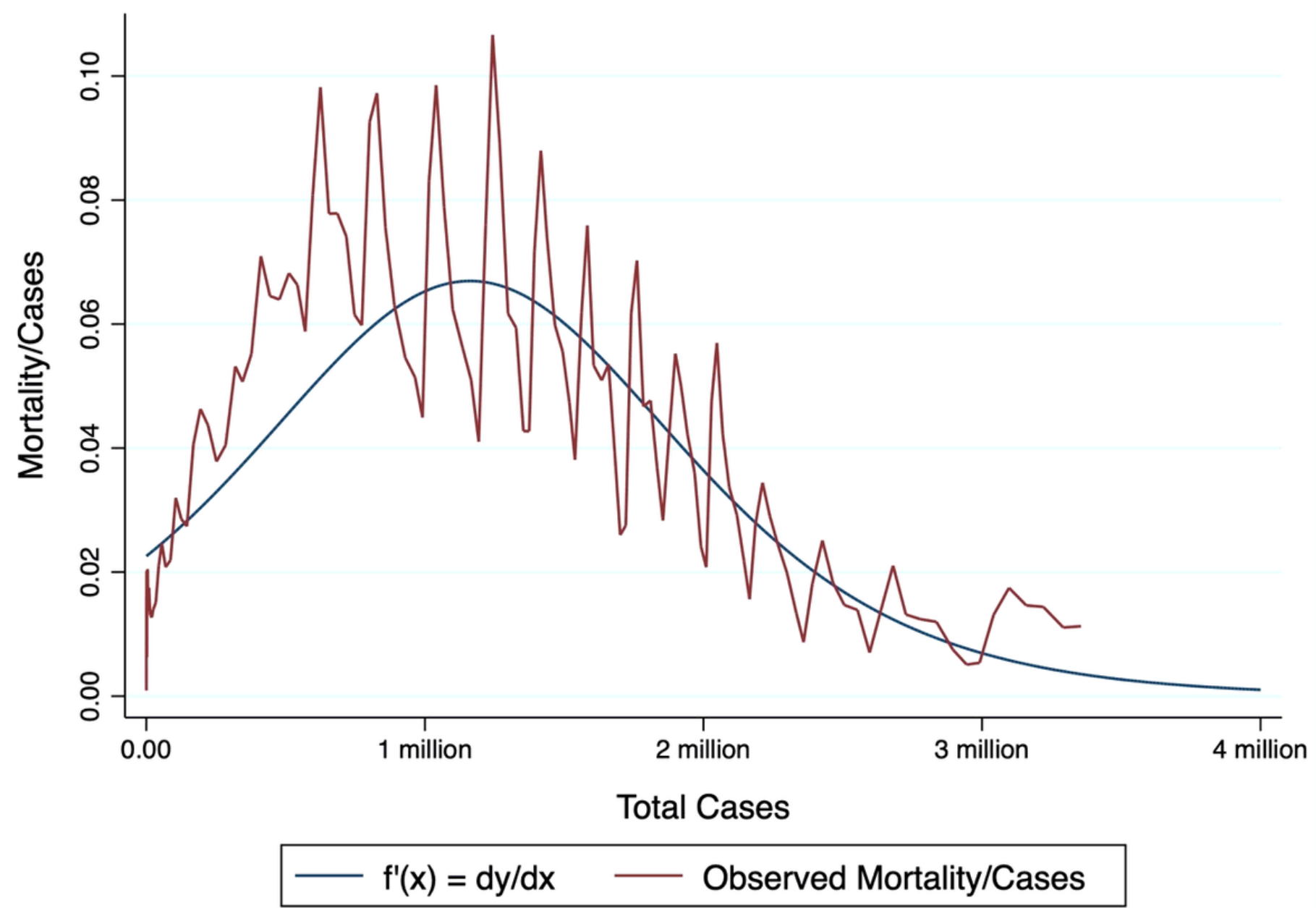

Figure 5

USA COVID-19 Daily Change in Mortality/Change in Cases: July 11, 2020 